PROCEEDINGS OF THE

AMERICAN MATHEMATICAL SOCIETY

Volume 138, Number 12, December 2010, Pages 4187-4195

S 0002-9939(2010)10441-0

Article electronically published on June 22, 2010

\title{
AN OCTONION ALGEBRA ORIGINATING IN COMBINATORICS
}

\author{
DRAGOMIR Ž. ĐOKOVIĆ AND KAIMING ZHAO
}

(Communicated by Birge Huisgen-Zimmermann)

\begin{abstract}
C.H. Yang discovered a polynomial version of the classical Lagrange identity expressing the product of two sums of four squares as another sum of four squares. He used it to give short proofs of some important theorems on composition of $\delta$-codes (now known as $T$-sequences). We investigate the possible new versions of his polynomial Lagrange identity. Our main result shows that all such identities are equivalent to each other.
\end{abstract}

\section{INTRODUCTION}

C.H. Yang [7, 8, 9] discovered a polynomial version (see below) of the classical Lagrange identity on the product of two sums of four squares. He used his identity to give short and elegant proofs of some important theorems 9 in combinatorics of binary and ternary sequences. These results provide new methods for the construction of several classes of combinatorial objects such as $T$-sequences, orthogonal designs, and Hadamard matrices [5, 9. Our motivation and the main goal was to investigate the possible new versions of the polynomial Lagrange identity. However, our main result shows that all such identities are equivalent to each other (for the precise statement, see Theorem 4.1).

Let $A=\mathbf{Z}\left[z, z^{-1}\right]$ be the Laurent polynomial ring over the integers $\mathbf{Z}$. For any $f=f(z)=\sum_{k} a_{k} z^{k} \in A, a_{k} \in \mathbf{Z}$, we define its conjugate as $f^{*}=f\left(z^{-1}\right)$. We also say that $a_{0}$ is the constant term of $f$ and write $\operatorname{CT}(f)=a_{0}$. Note that $\mathrm{CT}\left(f f^{*}\right)=\sum_{k} a_{k}^{2}$. Let $A_{0}$ be the fixed subring of $A$ under the conjugation, i.e., $A_{0}=\left\{f \in A \mid f=f^{*}\right\}$. It is easy to see that $A_{0}=\mathbf{Z}\left[z+z^{-1}\right]$. We embed the polynomial ring $\mathbf{Z}[t]$ into $A$ by sending $t \rightarrow z+z^{-1}$ and view $A$ as a $\mathbf{Z}[t]$-algebra. It is easy to check that $A=A_{0} \oplus A_{0} z$. Thus $A$ is a free $\mathbf{Z}[t]$-module of rank 2 .

We are mainly interested in the free $A$-module $E=A^{4}$. When viewed as a $\mathbf{Z}[t]$-module it is again free but now its rank is 8 . For any $x=\left(x_{0}, x_{1}, x_{2}, x_{3}\right) \in E$ we define its norm as $N(x)=\sum_{k} x_{k} x_{k}^{*}$. Thus $N: E \rightarrow A_{0}$ is a quadratic form on the $A_{0}$-module $E$.

Received by the editors May 12, 2009 and, in revised form, September 21, 2009 and February 13, 2010 .

2010 Mathematics Subject Classification. Primary 17A75, 05B20, 05 B30.

Key words and phrases. Laurent polynomial ring, polynomial Lagrange identity, octonion algebra, composition algebra.

The first author was supported by an NSERC Discovery Grant.

The second author was supported by the NSERC and the NSF of China (Grant 10871192).

(C)2010 American Mathematical Society Reverts to public domain 28 years from publication 
Now we state the Lagrange identity for Laurent polynomials (modified Theorem 1 in [9]). Let $x=\left(x_{k}\right), y=\left(y_{k}\right) \in E$ and define $(p, q, r, s) \in E$ by Yang's formulae:

$$
\begin{aligned}
p & =x_{0} y_{0}-x_{1} y_{1}^{*}-x_{2} y_{2}^{*}-x_{3} y_{3}^{*}, \\
q & =x_{0} y_{1}+x_{1} y_{0}^{*}+x_{2}^{*} y_{3}^{*}-x_{3}^{*} y_{2}^{*}, \\
r & =x_{0} y_{2}-x_{1}^{*} y_{3}^{*}+x_{2} y_{0}^{*}+x_{3}^{*} y_{1}^{*}, \\
s & =x_{0} y_{3}+x_{1}^{*} y_{2}^{*}-x_{2}^{*} y_{1}^{*}+x_{3} y_{0}^{*}
\end{aligned}
$$

We can use these formulae to define an $A_{0}$-bilinear multiplication "o" on $E$ by $x \circ y=(p, q, r, s)$. It is straightforward to verify that the Lagrange identity $N(x \circ y)=N(x) N(y)$ is indeed valid.

We shall see in the next section that $(E, \circ)$ is in fact an octonion algebra over $\mathbf{Z}[t]$. In Section 3 we give an explicit description of the orthogonal group $\mathrm{O}(N)$ of the pair $(E, N)$; see Theorem 3.4. In Section 4 we show that all $A_{0}$-bilinear multiplications on $E$ satisfying the Lagrange identity are equivalent, in the sense defined there, to the above multiplication "o".

Our main result can probably be generalized by replacing $\mathbf{Z}$ with a more general commutative ring. We decided not to pursue this here in order to preserve the essentially combinatorial flavor of the original problem.

We are grateful to the referee for correcting a couple of errors in the original proof of Lemma 4.3, for giving the stronger result in Theorem 4.3, and for other detailed comments and suggestions. Actually most of the proofs have been improved by the referee.

\section{YANG FORMULAE DEFINE AN OCTONION ALGEBRA}

We make $A=\mathbf{Z}\left[z, z^{-1}\right]$ into a $\mathbf{Z}[t]$-algebra via the homomorphism $\mathbf{Z}[t] \rightarrow A$ sending $t \rightarrow z+z^{-1}$. We shall often identify $\mathbf{Z}[t]$ with its image $A_{0}$ under this homomorphism. According to the definition in [1, Chapter III, $\S 2], A$ is a quadratic $\mathbf{Z}[t]$-algebra. By using the basis $\{1, z\}$, we see that its type is $(-1, t)$. Indeed we have $z^{2}=-1+t z$. Moreover, $(A, *)$ is an example of a Cayley algebra (see [1]).

Let $H=A \times A$, a free $A$-module of rank 2. We shall also view it as a free $\mathbf{Z}[t]$-module of rank 4 with basis $(1,0),(z, 0),(0,1),(0, z)$. We make $H$ into an associative noncommutative algebra by using the Cayley-Dickson process; i.e., we define the multiplication in $H$ by

$$
(a, b)(c, d)=\left(a c-d^{*} b, b c^{*}+d a\right), \quad a, b, c, d \in A .
$$

The involution "*" on $A$ extends to an involutory anti-automorphism of $H$ by setting

$$
(a, b)^{*}=\left(a^{*},-b\right), \quad a, b \in A .
$$

Thus $H$ is an example of a quaternion algebra over $\mathbf{Z}[t]$; see [1, No. 5, Example 2 . As such, it has type $(-1, t,-1)$.

Let $E=H \times H$, a free $A$-module of rank 4 . We shall also view it as a free $\mathbf{Z}[t]$-module of rank 8 with basis

$$
\begin{array}{ll}
e_{0}=(1,0,0,0), & e_{0}^{\prime}=z e_{0}=(z, 0,0,0), \\
e_{1}=(0,1,0,0), & e_{1}^{\prime}=z e_{1}=(0, z, 0,0), \\
e_{2}=(0,0,1,0), & e_{2}^{\prime}=z e_{2}=(0,0, z, 0), \\
e_{3}=(0,0,0,1), & e_{3}^{\prime}=z e_{3}=(0,0,0, z) .
\end{array}
$$


We make $E$ into a nonassociative algebra by using the Cayley-Dickson process once more. Thus, we define the multiplication in $E$ by

$$
(u, v)(x, y)=\left(u x-y^{*} v, v x^{*}+y u\right), \quad u, v, x, y \in H .
$$

The involution "*" on $H$ extends to one on $E$ by setting

$$
(u, v)^{*}=\left(u^{*},-v\right), \quad u, v \in H .
$$

Thus $E$ is an example of an octonion algebra over $\mathbf{Z}[t]$; see [1, Chapter III, Appendix]. By using the above basis, we find that the type of this octonion algebra is $(-1, t,-1,-1)$.

Let us write the above octonion multiplication in terms of the $A$-basis $\left\{e_{0}, e_{1}\right.$, $\left.e_{2}, e_{3}\right\}$. For $x=\left(x_{0}, x_{1}, x_{2}, x_{3}\right)$ and $y=\left(y_{0}, y_{1}, y_{2}, y_{3}\right)$, we find that

$$
\begin{aligned}
x y= & \left(x_{0} y_{0}-x_{1} y_{1}^{*}-x_{2} y_{2}^{*}-x_{3}^{*} y_{3},\right. \\
& x_{0} y_{1}+x_{1} y_{0}^{*}+x_{2}^{*} y_{3}-x_{3} y_{2}^{*}, \\
& x_{0} y_{2}-x_{1}^{*} y_{3}+x_{2} y_{0}^{*}+x_{3} y_{1}^{*}, \\
& \left.x_{0}^{*} y_{3}+x_{1} y_{2}-x_{2} y_{1}+x_{3} y_{0}\right) .
\end{aligned}
$$

Hence, the map sending $\left(x_{0}, x_{1}, x_{2}, x_{3}\right) \rightarrow\left(x_{0}, x_{1}, x_{2}, x_{3}^{*}\right)$ is an isomorphism of this octonion algebra with the algebra $(E, \circ)$ defined by the Yang formulae.

\section{ORTHOGONAL GROUP}

It is obvious that $U_{1}=\left\{x \in A \mid x x^{*}=1\right\}$ is the group of invertible elements of $A$. It consists of the elements $\pm z^{k}, k \in \mathbf{Z}$. In our proofs below we shall often use the following obvious fact: The subset $U_{1}$ generates $A$ as an additive group.

The ring homomorphisms $\varphi: A \rightarrow \mathbf{C}$ are parametrized by nonzero complex numbers $w$. By definition, the homomorphism $\varphi_{w}$ corresponding to $w$ sends the indeterminate $z$ to $w$. The homomorphism $\varphi_{w}$ is compatible with the involutions (conjugation on $A$ and complex conjugation on $\mathbf{C}$ ); i.e., $\varphi_{w}\left(x^{*}\right)=\overline{\varphi_{w}(x)}$ for all $x \in A$ if and only if $|w|=1$.

The following lemma will be used in the next section.

Lemma 3.1. If $m \in \mathbf{Z}$ is not a square, then the equation $x x^{*}=m$ has no solution in $A$.

Proof. Assume that $f f^{*}=m$ for some $f \in A$. By applying the homomorphism $\varphi_{1}$ we obtain $f(1) f^{*}(1)=m$. As $f^{*}(1)=f(1) \in \mathbf{Z}$, we have a contradiction.

We also remark that, for $x \in A, \mathrm{CT}\left(x x^{*}\right)=0$ implies $x=0$, and $\mathrm{CT}\left(x x^{*}\right)=1$ implies $x x^{*}=1$.

Denote the general linear group of the $A_{0}$-module $E$ by GL $\left(A_{0}, E\right)$. We introduce the orthogonal groups

$$
\begin{aligned}
\mathrm{O}(N) & =\left\{\varphi \in \mathrm{GL}\left(A_{0}, E\right) \mid N(\varphi(u))=N(u), \forall u \in E\right\}, \\
\mathrm{O}_{4}(\mathbf{Z}) & =\left\{\varphi \in \mathrm{GL}_{4}(\mathbf{Z}) \mid N(\varphi(u))=N(u), \forall u \in \mathbf{Z}^{4}\right\},
\end{aligned}
$$

where $\mathbf{Z}^{4}$ is considered as an additive subgroup of $E=A^{4}$.

Let $Q: E \times E \rightarrow A_{0}$ be the polar form of $N$, i.e.,

$$
\begin{aligned}
Q(x, y) & =N(x+y)-N(x)-N(y) \\
& =\sum_{k=0}^{3}\left(x_{k}^{*} y_{k}+x_{k} y_{k}^{*}\right) .
\end{aligned}
$$


Note that $Q$ is a symmetric $A_{0}$-bilinear $\mathrm{O}(N)$-invariant form and that $Q$ is nondegenerate; i.e., its kernel is 0 .

Let us begin with a useful remark.

Remark 3.2. An $A_{0}$-linear map $\varphi: E \rightarrow E$ preserving $N$ is automatically bijective, and so $\varphi \in \mathrm{O}(N)$. Indeed, as $Q$ is nondegenerate, the injectivity is obvious. If $S$ is the matrix of $Q$ with respect to some $A_{0}$-basis of $E$, then $\operatorname{det}(\varphi)^{2} \operatorname{det}(S)=$ $\operatorname{det}(S) \neq 0$, forcing $\operatorname{det}(\varphi)= \pm 1$, and $\varphi$ is bijective.

The following result is crucial to this paper.

Theorem 3.3. (a) The group $\mathrm{O}_{4}(\mathbf{Z})$ consists of entry permutations on $\mathbf{Z}^{4}$ with arbitrary sign changes.

(b) The set $U_{1}^{\prime}=\left\{x \in A \mid x x^{*}=-\left(z-z^{-1}\right)^{2}\right\}$ is equal to $\left(z-z^{-1}\right) U_{1}$.

(c) The unit sphere $U_{4}=\{x \in E \mid N(x)=1\}$ is equal to $\bigcup_{k=0}^{3} U_{1} e_{k}$.

(d) The sphere $U_{4}^{\prime}=\left\{x \in E \mid N(x)=-\left(z-z^{-1}\right)^{2}\right\}$ is equal to $\left(z-z^{-1}\right) U_{4}$.

Proof. (a) Fix $\alpha \in \mathrm{O}_{4}(\mathbf{Z})$. Let $\alpha\left(e_{i}\right)=\sum_{j} a_{i j} e_{j}$, where $a_{i j} \in \mathbf{Z}$. From $1=$ $N\left(\alpha\left(e_{i}\right)\right)=\sum_{j} a_{i j}^{2}$ we see that, for each $i$, exactly one of $a_{i j}$ is \pm 1 and the other ones vanish. The rest follows easily.

(b) By applying the homomorphisms $\varphi_{ \pm 1}$ to $x x^{*}=-\left(z-z^{-1}\right)^{2}$, we conclude that $x$ is divisible by $z-1$ and $z+1$, and so we have $x=\left(z-z^{-1}\right) y$ for some $y \in A$. As $x x^{*}=-\left(z-z^{-1}\right)^{2} y y^{*}$, we deduce that $y y^{*}=1$, i.e., $y \in U_{1}$.

(c) Suppose $x=\left(x_{0}, x_{1}, x_{2}, x_{3}\right) \in U_{4}$. Then $\sum_{k=0}^{3} x_{k} x_{k}^{*}=1$. By comparing the constant terms, we conclude that exactly one of the $x_{k}$ belongs to $U_{1}$ while the others vanish.

(d) Suppose $x=\left(x_{k}\right) \in U_{4}^{\prime}$. Then $\sum_{k} x_{k} x_{k}^{*}=2-z^{2}-z^{-2}$. Hence $\sum_{k} \mathrm{CT}\left(x_{k} x_{k}^{*}\right)$ $=2$, which by the above remark implies that exactly one of the $x_{k} \mathrm{~s}$ is nonzero. This nonzero component belongs to $\left(z-z^{-1}\right) U_{1}$ by (b).

Note that $\mathrm{O}_{4}(\mathbf{Z})=E_{16} \Sigma_{4}$ is the semidirect product of the elementary abelian group $E_{16}$ of order 16 which acts on $\mathbf{Z}^{4}$ by multiplying the coordinates with \pm 1 and the symmetric group $\Sigma_{4}$ of degree 4 which permutes the coordinates.

For $u=\left(u_{0}, u_{1}, u_{2}, u_{3}\right) \in U_{1}^{4}$ we define $\sigma_{u} \in \mathrm{O}(N)$ by $\sigma_{u}(x)=\left(u_{k} x_{k}\right)$, where $x=\left(x_{k}\right) \in E$. Then

$$
T=\left\{\sigma_{u} \mid u \in U_{1}^{4}\right\}
$$

is an abelian subgroup of $\mathrm{O}(N)$.

Any $\alpha \in \mathrm{O}_{4}(\mathbf{Z})$ extends uniquely to an $A$-linear element of $\mathrm{O}(N)$, still denoted by $\alpha$. Thus we consider $\mathrm{O}_{4}(\mathbf{Z})$ as a subgroup of $\mathrm{O}(N)$. Define $\tau_{0} \in \mathrm{O}(N)$ by $\tau_{0}\left(x_{0}, x_{1}, x_{2}, x_{3}\right)=\left(x_{0}^{*}, x_{1}, x_{2}, x_{3}\right)$, and define similarly $\tau_{k}$ for $k=1,2,3$. Let $\Gamma$ denote the group of order 16 generated by the $\tau_{k}$ s. The group $\mathrm{O}(N)$ admits the following factorization.

Theorem 3.4. $\mathrm{O}(N)=T \Sigma_{4} \Gamma$.

Proof. Let $\varphi \in \mathrm{O}(N)$. Since $\varphi\left(U_{4}\right)=U_{4}$, for any index $i$ there exist $u_{i} \in U_{1}$ and an index $i^{\prime}$ such that $\varphi\left(e_{i}\right)=u_{i} e_{i^{\prime}}$. Since the form $Q$ is $\varphi$-invariant, the map $i \rightarrow i^{\prime}$ must be a permutation of $\{0,1,2,3\}$.

For any $a \in A$ we have $a+a^{*} \in A_{0}$ and so

$$
\varphi\left(\left(a+a^{*}\right) e_{i}\right)=\left(a+a^{*}\right) u_{i} e_{i^{\prime}} .
$$


Now let $a \in U_{1}, a \neq \pm 1$. Then each of the terms $\varphi\left(a e_{i}\right), \varphi\left(a^{*} e_{i}\right), a u_{i} e_{i^{\prime}}$ and $a^{*} u_{i} e_{i^{\prime}}$ belongs to $U_{4}$, and the sum of the first two terms is not 0 . It follows that $\varphi\left(a e_{i}\right)$ is equal to $a u_{i} e_{i^{\prime}}$ or $a^{*} u_{i} e_{i^{\prime}}$.

Assume that there exist $a, b \in U_{1}$ different from \pm 1 such that $\varphi\left(a e_{i}\right)=a u_{i} e_{i^{\prime}}$ while $\varphi\left(b e_{i}\right)=b^{*} u_{i} e_{i^{\prime}}$. Then $\varphi\left((a+b) e_{i}\right)=\left(a+b^{*}\right) u_{i} e_{i^{\prime}}$, and by taking the norms we obtain the contradiction $\left(a-a^{*}\right)\left(b-b^{*}\right)=0$. Consequently, one of the two identities, $\varphi\left(a e_{i}\right)=a u_{i} e_{i^{\prime}}$ or $\varphi\left(a e_{i}\right)=a^{*} u_{i} e_{i^{\prime}}$, must hold for all $a \in A$. Equivalently, there exists $\varepsilon_{i} \in\{0,1\}$ such that $\varphi \tau_{i}^{\varepsilon_{i}}\left(a e_{i}\right)=a u_{i} e_{i^{\prime}}$ for all $a \in A$. Let

$$
u=\left(u_{i}\right) \in U_{1}^{4} \quad \text { and } \quad \tau=\prod_{i=0}^{3} \tau_{i}^{\varepsilon_{i}} \in \Gamma .
$$

The composite $\beta=\sigma_{u}^{-1} \varphi \tau$ is $A$-linear, and we have $\sigma_{u}^{-1} \varphi \tau\left(e_{i}\right)=e_{i^{\prime}}$ for all $i$. Hence, $\varphi=\sigma_{u} \beta \tau$ with $\sigma_{u} \in T, \beta \in \Sigma_{4}$ and $\tau \in \Gamma$.

\section{Composition algebra structures on $E$}

In this section we determine all $A_{0}$-bilinear multiplications "." on $E$ which satisfy the (polynomial) Lagrange identity

$$
N(x \cdot y)=N(x) N(y) .
$$

Since $E$ is a free $A_{0}$-module of rank 8 and the form $Q$ is nondegenerate, such an algebra $(E, \cdot)$ will be a composition algebra in the sense of the definition in 3 , p. 305] provided that it has an identity element.

We say that two $A_{0}$-bilinear multiplications $\star$ and $\diamond$ are equivalent if there exist $\sigma_{1}, \sigma_{2}, \tau \in \mathrm{O}(N)$ such that $x \diamond y=\tau\left(\sigma_{1}(x) \star \sigma_{2}(y)\right)$ for all $x, y \in E$.

The following theorem is our main result.

Theorem 4.1. Any $A_{0}$-bilinear multiplication ". on E satisfying the Lagrange identity (4.1) is equivalent to the multiplication "o" defined by Yang's formulae.

Proof. We shall reduce the proof to the special case where $e_{0}$ is the identity element of the $A_{0}$-algebra $(E, \cdot)$. Then the assertion of the theorem follows from the next theorem.

Define $A_{0}$-linear maps $L, R: E \rightarrow E$ by $L(u)=e_{0} \cdot u$ and $R(u)=u \cdot e_{0}$. The Lagrange identity implies that $L$ and $R$ preserve $N$. By Remark 3.2, $L, R \in \mathrm{O}(N)$.

Next we use a well known argument due to, at least, Kaplansky [2]. We have $N\left(R^{-1}(x) \cdot L^{-1}(y)\right)=N(x) N(y)$ for $x, y \in E$. With $x \star y=R^{-1}(x) \cdot L^{-1}(y)$, we have

$$
\begin{aligned}
& \left(e_{0} \cdot e_{0}\right) \star x=R^{-1}\left(e_{0} \cdot e_{0}\right) \cdot L^{-1}(x)=e_{0} \cdot L^{-1}(x)=x, \\
& x \star\left(e_{0} \cdot e_{0}\right)=R^{-1}(x) \cdot L^{-1}\left(e_{0} \cdot e_{0}\right)=R^{-1}(x) \cdot e_{0}=x .
\end{aligned}
$$

Thus $e_{0} \cdot e_{0}$ is the identity element of the algebra $(E, \star)$. As $e_{0} \cdot e_{0} \in U_{4}$, we have $e_{0} \cdot e_{0}=a e_{i}$ for some $a \in U_{1}$ and some index $i$. Hence, there exists $\sigma \in \mathrm{O}(N)$ such that $\sigma\left(e_{0}\right)=e_{0} \cdot e_{0}$. If $x \diamond y=\sigma^{-1}(\sigma(x) \star \sigma(y))$, then $e_{0}$ is the identity element of the algebra $(A, \diamond)$.

We assume now that $e_{0}$ is the identity element of $(E, \cdot)$. Consequently, this is a composition algebra in which the alternative laws

$$
x \cdot(x \cdot y)=(x \cdot x) \cdot y, \quad(x \cdot y) \cdot y=x \cdot(y \cdot y)
$$


are valid [3, p. 306]. The reader should consult this book for additional properties of composition algebras. We state only a few properties that we need.

Define the $A_{0}$-linear map trace $T: E \rightarrow A_{0}$ by $T(x)=Q\left(e_{0}, x\right)$. Then for any $x \in E$ we have $x \cdot x-T(x) x+N(x) e_{0}=0$. Linearizing gives

$$
x \cdot y+y \cdot x=T(x) y+T(y) x-Q(x, y) e_{0}, \forall x, y \in E .
$$

Clearly $A e_{0} \perp A^{3}$ with respect to $Q$, where $A^{3}=A e_{1}+A e_{2}+A e_{3}$.

Recall that we have extended the conjugation * from $A$ to $E$ in Section 2 , Moreover, note that

$$
\left(a e_{0}+v\right)^{*}=a^{*} e_{0}-v, \quad a \in A, v \in A^{3} .
$$

It is easy to verify that

$$
Q\left(x, z \cdot y^{*}\right)=Q(x \cdot y, z)=Q\left(y, x^{*} \cdot z\right), \forall x, y, z \in E .
$$

The fact that in the next theorem one can assert that the two algebras are actually isomorphic is due to the referee.

Theorem 4.2. Every $A_{0}$-algebra $(E, \cdot)$ satisfying the Lagrange identity (4.1) and having $e_{0}$ as the identity element is isomorphic to the Yang algebra $(E, \circ)$.

We shall break the proof into several lemmas.

Lemma 4.3. By replacing "." with an isomorphic multiplication, we may also assume that

$$
\left(a e_{0}\right) \cdot y=a y
$$

for all $a \in A$ and $y \in E$.

Proof. Let us fix an index $i$ and $b \in U_{1}$. Then the right multiplication by $b e_{i}$ belongs to $O(N)$, and Theorem 3.4 yields an index $j$ as well as $u_{j} \in U_{1}$ such that $\left(a e_{0}\right) \cdot\left(b e_{i}\right)=u_{j} a e_{j}$ for all $a \in A$ or $\left(a e_{0}\right) \cdot\left(b e_{i}\right)=u_{j} a^{*} e_{j}$ for all $a \in A$. Specializing $a$ to 1 implies that $j=i$ and $u_{j}=b$; hence $\left(a e_{0}\right) \cdot\left(b e_{i}\right)=a b e_{i}$ for all $a \in A$ or $\left(a e_{0}\right) \cdot\left(b e_{i}\right)=a^{*} b e_{i}$ for all $a \in A$.

Assume that $\left(a e_{0}\right) \cdot\left(b_{1} e_{i}\right)=a b_{1} e_{i}$ holds for all $a \in U_{1}$ and $\left(a e_{0}\right) \cdot\left(b_{2} e_{i}\right)=$ $a^{*} b_{2} e_{i}$ holds for all $a \in U_{1}$, for some units $b_{1}, b_{2}$ different from \pm 1 . Then $\left(a e_{0}\right)$. $\left(\left(b_{1}+b_{2}\right) e_{i}\right)=\left(a b_{1}+a^{*} b_{2}\right) e_{i}$ holds for all $a \in U_{1}$. By taking norms on both sides, we obtain that

$$
\left(1-a^{2}\right) b_{1} b_{2}^{*}+\left(1-\left(a^{*}\right)^{2}\right) b_{1}^{*} b_{2}=0
$$

for all $a \in U_{1}$. This is clearly a contradiction.

Since $U_{1}$ generates $A$ as an abelian group, we conclude that either $\left(a e_{0}\right) \cdot\left(b e_{i}\right)=$ $a b e_{i}$ for all $a, b \in A$ or $\left(a e_{0}\right) \cdot\left(b e_{i}\right)=a^{*} b e_{i}$ for all $a, b \in A$. In particular, $\left(a e_{0}\right) \cdot\left(b e_{i}\right)$ is $A$-linear in $b$.

If $\left(a e_{0}\right) \cdot\left(b e_{0}\right)=a^{*} b e_{0}$ for all $a, b \in A$, then setting $b=1$ gives the contradiction: $a e_{0}=a^{*} e_{0}$ for all $a \in A$. Thus we have $\left(a e_{0}\right) \cdot\left(b e_{0}\right)=a b e_{0}$ for all $a, b \in A$. For $i \neq 0$, there is an $\varepsilon_{i} \in\{0,1\}$ such that $\tau_{i}^{\varepsilon_{i}}\left(\tau_{i}^{\varepsilon_{i}}\left(a e_{0}\right) \cdot \tau_{i}^{\varepsilon_{i}}\left(b e_{i}\right)\right)=a b e_{i}$ for all $a, b \in A$.

If $\tau$ is the product of the $\tau_{i}^{\varepsilon_{i}}$ with $i \neq 0$, then $\tau^{2}=1$ and the multiplication " $\star$ " defined by $x \star y=\tau(\tau(x) \cdot \tau(y))$ satisfies all the requirements.

Moreover, the map $\tau:(E, \star) \rightarrow(E, \cdot)$ is an isomorphism of unital $A_{0}$-algebras. 
We assume from now on that the identity (4.5) holds. This property will be shared by the modified multiplication, which will be introduced in Lemma 4.6 .

Lemma 4.4. For $a \in A$ and $x \in A e_{1}+A e_{2}+A e_{3}$, we have $x \cdot\left(a e_{0}\right)=a^{*} x$.

Proof. Recall that $A e_{1}+A e_{2}+A e_{3}$ is the kernel of the trace $T$ and is perpendicular to $A e_{0}$ relative to $Q$. By using (4.3), we obtain that

$$
\begin{aligned}
x \cdot\left(a e_{0}\right) & =x \cdot\left(a e_{0}\right)+\left(a e_{0}\right) \cdot x-\left(a e_{0}\right) \cdot x \\
& =T\left(a e_{0}\right) x+T(x) a e_{0}-Q\left(x, a e_{0}\right) e_{0}-a x \\
& =\left(a+a^{*}-a\right) x=a^{*} x .
\end{aligned}
$$

Lemma 4.5. For $a, b \in A$ and $i \neq 0$ we have $\left(a e_{i}\right) \cdot\left(b e_{i}\right)=-a b^{*} e_{0}$.

Proof. Since $N$ is anisotropic, it suffices to show that $N\left(\left(a e_{i}\right) \cdot\left(b e_{i}\right)+a b^{*} e_{0}\right)=0$. Expanding the LHS and applying (4.4) gives

$$
\begin{aligned}
N\left(\left(a e_{i}\right) \cdot\left(b e_{i}\right)+a b^{*} e_{0}\right) & =2 N(a) N(b)+Q\left(\left(a e_{i}\right) \cdot\left(b e_{i}\right), a b^{*} e_{0}\right) \\
& =2 N(a) N(b)+Q\left(a e_{i},\left(a b^{*} e_{0}\right) \cdot\left(b e_{i}\right)^{*}\right) \\
& =2 N(a) N(b)-Q\left(a e_{i},\left(a b^{*} e_{0}\right) \cdot\left(b e_{i}\right)\right) \\
& =2 N(a) N(b)-N(b) Q\left(a e_{i}, a e_{i}\right) \\
& =2 N(a) N(b)-2 N(a) N(b)=0 .
\end{aligned}
$$

Lemma 4.6. By replacing ". with an isomorphic multiplication, we may assume that

$$
e_{i} \cdot e_{j}=e_{k}=-e_{j} \cdot e_{i}
$$

for any cyclic permutation $(i, j, k)$ of $(1,2,3)$.

Proof. Let $(i, j, k)$ be a cyclic permutation of $(1,2,3)$. Since $\left(e_{0}+e_{i}\right) \cdot\left(e_{0}+e_{j}\right)=$ $e_{0}+e_{i}+e_{j}+e_{i} \cdot e_{j}$ and $N\left(e_{0}+e_{j}\right)=N\left(e_{0}+e_{j}\right)=2$, we have $N\left(e_{0}+e_{i}+e_{j}+e_{i} \cdot e_{j}\right)=4$. As $e_{i} \cdot e_{j} \in U_{4}$, Lemma 3.1 implies that $e_{i} \cdot e_{j} \in U_{1} e_{k}$. A similar argument shows that $e_{j} \cdot e_{i} \in U_{1} e_{k}$.

In particular we have $e_{1} \cdot e_{2}=u e_{3}$ for some $u \in U_{1}$. The multiplication " $\star$ " defined by $x \star y=\sigma_{a}^{-1}\left(\sigma_{a}(x) \cdot \sigma_{a}(y)\right)$, where $a=(1,1,1, u)$, has the previously stated property and sends $\left(e_{1}, e_{2}\right) \rightarrow e_{3}$. By replacing "." with this new multiplication, we may assume that $e_{1} \cdot e_{2}=e_{3}$.

Since $\left(e_{i}+e_{j}\right) \cdot\left(e_{i}+e_{j}\right)=e_{i} \cdot e_{j}+e_{j} \cdot e_{i}-2 e_{0}$, we have $N\left(e_{i} \cdot e_{j}+e_{j} \cdot e_{i}-2 e_{0}\right)=4$. Since $e_{i} \cdot e_{j}+e_{j} \cdot e_{i} \in A e_{k}$, it follows that this sum is 0 , i.e., $e_{j} \cdot e_{i}=-e_{i} \cdot e_{j}$. In particular, we have $e_{2} \cdot e_{1}=-e_{3}$. Thus the assertion of the lemma is valid if $(i, j, k)=(1,2,3)$.

The remaining equalities (4.6) follow easily by using the alternative laws (4.2). For instance, we have $e_{1} \cdot e_{3}=e_{1} \cdot\left(e_{1} \cdot e_{2}\right)=\left(e_{1} \cdot e_{1}\right) \cdot e_{2}=\left(-e_{0}\right) \cdot e_{2}=-e_{2}$.

To finish the proof, we point out that the map $\sigma_{a}:(E, \star) \rightarrow(E, \cdot)$ is an isomorphism of unital $A_{0}$-algebras.

In view of the last lemma, we may assume now (and we do) that the identities (4.6) are valid for any cyclic permutation $(i, j, k)$ of $(1,2,3)$. 
Lemma 4.7. For any cyclic permutation $(i, j, k)$ of $(1,2,3)$ we have

$$
\left(a e_{i}\right) \cdot\left(b e_{j}\right)=a^{*} b^{*} e_{k}=-\left(b e_{j}\right) \cdot\left(a e_{i}\right)
$$

for all $a, b \in A$. Consequently, the multiplications " " and "o" coincide.

Proof. In view of (4.3), it suffices to prove the first equality. We first treat the case

$a=1$. By applying the linearized left alternative law, we find that

$$
\begin{aligned}
e_{i} \cdot\left(b e_{j}\right) & =e_{i} \cdot\left(\left(b e_{0}\right) \cdot e_{j}\right) \\
& =\left(e_{i} \cdot\left(b e_{0}\right)+\left(b e_{0}\right) \cdot e_{i}\right) \cdot e_{j}-\left(b e_{0}\right) \cdot\left(e_{i} \cdot e_{j}\right) \\
& =\left(b+b^{*}\right) e_{k}-b e_{k}=b^{*} e_{k} .
\end{aligned}
$$

For arbitrary $a$, by using this special case, the linearized right alternative law and Lemma 4.4, we obtain that

$$
\begin{aligned}
\left(a e_{i}\right) \cdot\left(b e_{j}\right) & =\left(e_{i} \cdot\left(a^{*} e_{0}\right)\right) \cdot\left(b e_{j}\right) \\
& =e_{i} \cdot\left(\left(a^{*} e_{0}\right) \cdot\left(b e_{j}\right)+\left(b e_{j}\right) \cdot\left(a^{*} e_{0}\right)\right)-\left(e_{i} \cdot\left(b e_{j}\right)\right) \cdot\left(a^{*} e_{0}\right) \\
& =e_{i} \cdot\left(\left(a+a^{*}\right) b e_{j}\right)-\left(b^{*} e_{k}\right) \cdot\left(a^{*} e_{0}\right) \\
& =\left(\left(a+a^{*}\right) b^{*}-a b^{*}\right) e_{k}=a^{*} b^{*} e_{k} .
\end{aligned}
$$

This concludes the proof of Theorem 4.2 and also of Theorem 4.1

The referee supplied an alternative proof of Theorem 4.2 His proof proceeds first by changing scalars from the base ring $\mathbf{Z}[t]$ to its quotient field $\mathbf{Q}(t)$, and then applying a theorem of Thakur [6] (see also [4, Chapter VIII, Exercise 6]) which establishes a connection between octonion algebras and ternary Hermitian forms. In the case of a Yang algebra, let us write $E=A e_{0} \oplus A^{3}$, where $A^{3}$ is the column $A$ space. Extending the conjugation of $A$ componentwise to $A^{3}$, we have a Hermitian form $h: A^{3} \times A^{3} \rightarrow A$ given by $h(v, w)=v^{t} w^{*}$. With this notation, the Yang multiplication is given by

$$
\left(a e_{0} \oplus v\right) \circ\left(b e_{0} \oplus w\right)=(a b-h(v, w)) e_{0} \oplus\left(a w+b^{*} v+v^{*} \times w^{*}\right),
$$

where $a, b \in A$ and $v, w \in A^{3}$. The sign $\times$ stands for the ordinary cross product in 3 -space.

\section{REFERENCES}

[1] N. Bourbaki, Algèbre, Chapitres 1 à 3, Hermann, Paris, 1970. MR0274237 (43:2)

[2] I. Kaplansky, Infinite-dimensional quadratic forms admitting composition, Proc. Amer. Math. Soc. 4 (1953), 956-960. MR0059895(15:596g)

[3] M.-A. Knus, Quadratic and Hermitian Forms over Rings, Grundlehren der mathematischen Wissenschaften 294, Springer-Verlag, 1991. MR1096299 (92i:11039)

[4] M.-A. Knus, A. Merkurjev, M. Rost and J.-P. Tignol, The Book of Involutions, Amer. Math. Soc. Colloq. Publ. 44, Amer. Math. Soc., Providence, RI, 1998. MR.1632779 (2000a:16031)

[5] J. Seberry and M. Yamada, Hadamard matrices, sequences and block designs, in Contemporary Design Theory: A Collection of Surveys, Eds. J.H. Dinitz and D.R. Stinson, J. Wiley, New York, 1992, pp. 431-560. MR.1178508

[6] M. L. Thakur, Cayley algebra bundles on $\mathbf{A}_{K}^{2}$ revisited, Comm. Algebra 23 (1995), no. 13, 5119-5130. MR1356125(97a:17006)

[7] C. H. Yang, Lagrange identity for polynomials and $\delta$-codes of lengths $7 t$ and $13 t$, Proc. Amer. Math. Soc. 88 (1983), 746-750. MR702312 (85f:05034) 
[8] C. H. Yang, A composition theorem for $\delta$-codes, Proc. Amer. Math. Soc. 89 (1983), 375-378. MR712655 (85i:94025)

[9] C. H. Yang, On composition of four-symbol $\delta$-codes and Hadamard matrices, Proc. Amer. Math. Soc. 107 (1989), 763-776. MR979054 (90e:94043)

Department of Pure Mathematics, University of Waterloo, Waterloo, Ontario, N2L 3G1, CANADA

E-mail address: djokovic@uwaterloo.ca

Department of Mathematics, Wilfrid Laurier University, Waterloo, Ontario, N2L 3C5, Canada - And- Institute of Mathematics, Academy of Mathematics and System Sciences, Chinese Academy of Sciences, Beijing 100190, People's Republic of China

E-mail address: kzhao@wlu.ca 